\title{
O CURRÍCULO DO CURSO DE ENGENHARIA CIVIL CENTRADO NA APRENDIZAGEM: A MATRIZ POR COMPETÊNCIAS COMO POSSIBILIDADE DE INTEGRAÇÃO
}

Viviane Chulek-vivianechulek@utfpr.edu.br

Universidade Tecnológica Federal do Paraná, Departamento de Educação

Av. Prof ${ }^{a}$ Laura de Pacheco Bastos, 800

85.053-525 - Guarapuava - Paraná

Joice Cristine Kuritza - joicekuritza@utfpr.edu.br

Universidade Tecnológica Federal do Paraná, COECI

Av. Prof ${ }^{a}$ Laura de Pacheco Bastos, 800

85.053-525 - Guarapuava - Paraná

Mariane Kempka - marianekempka@utfpr.edu.br

Universidade Tecnológica Federal do Paraná, COECI

Av. Prof Laura de Pacheco Bastos, 800

85.053-525 - Guarapuava - Paraná

Ruth Nascimento Ferreira_ruth.nas@gmail.com

Universidade Tecnológica Federal do Paraná, COECI

Av. Prof ${ }^{a}$ Laura de Pacheco Bastos, 800

85.053-525 - Guarapuava - Paraná

Carlos Francisco Pecapedra Souza - pecapedra@utfpr.edu.br

Universidade Tecnológica Federal do Paraná, COECI

Av. Prof ${ }^{a}$ Laura de Pacheco Bastos, 800

85.053-525 - Guarapuava - Paraná

Resumo: Após a publicação das Diretrizes Curriculares Nacionais (DCN) dos cursos de engenharia, no ano de 2019, as instituições de ensino superior (IES) buscaram se adequar à nova organização. Para a reformulação dos projetos pedagógicos dos cursos de engenharia, a Universidade Tecnológica Federal do Paraná (UTFPR), por meio do Departamento de Educação, organizou uma Oficina de Design de Curso. O objetivo desta oficina foi desenvolver os projetos pedagógicos de forma a assegurar o desenvolvimento das competências necessárias ao profissional de engenharia. Com o processo de capacitação, o Núcleo Docente Estruturante (NDE) e os professores do curso de Engenharia Civil, campus Guarapuava, construíram uma nova proposta curricular, de maneira a garantir a formação técnica e o desenvolvimento das competências gerais e específicas. Os professores trabalharam de forma coletiva na revisão de todas as disciplinas do curso buscando adequálas ao modelo de aprendizagem dos três saberes: saber (conhecimento), saber-fazer (conhecimento técnico) e o saber-ser (atitude). Esta revisão iniciou pela "transformação" dos conteúdos em temas de estudo (TE), os quais se referem aos conhecimentos, deixando-os mais atrativos ao estudante. Dos TEs, o grupo elaborou os resultados de aprendizagem (RA), que interligam os três saberes à construção do perfil do egresso. Neste trabalho, apresentamse as competências e os elementos necessários à formação do perfil do egresso do curso de Engenharia Civil da UTFPR, campus Guarapuava, além de apresentar os TEs e RAs da disciplina de Cálculo I como exemplo.

Palavras-chave: Ensino por Competências. Engenharia Civil. Cálculo. 


\title{
1 INTRODUÇÃO
}

A UTFPR, por ser uma instituição multicampi, observou a necessidade de avaliação e acompanhamentos dos currículos dos cursos de graduação nos diferentes campus. Nesse sentido, a partir de fevereiro de 2017, foram realizados os fóruns das Disciplinas do Núcleo Básico (For-Bas), das Engenharias (FORENGE) e ainda os fóruns das licenciaturas (FORLIC) e das Tecnologias (FORTEC) com representação dos professores de todos os câmpus para delinear premissas comuns aos cursos de graduação respeitando as demandas regionais de cada câmpus.

A publicação das novas Diretrizes Curriculares pelo MEC, resolução no 2, de 24 de abril de 2019, que tinha como objetivos elevar a qualidade do ensino em Engenharia no país, permitir maior flexibilidade na estruturação dos cursos de forma a permitir a inovação curricular, reduzir a taxa de evasão e ainda direcionar para o atendimento de demandas futuras quando a formação de engenheiros (PARECER CNE/CES No: 1/2019), impõe a necessidade não apenas de revisão curricular mas de mudança de concepção sobre a formação de engenheiros na UTFPR.

A mudança no paradigma pauta o processo de revisão curricular na UTFPR e, está expressa em seu Projeto Pedagógico Institucional (PPI) que assume o compromisso de romper com a dualidade entre teoria e prática assumindo as duas dimensões como indissociáveis para a educação integral que não admite a separação entre as funções intelectuais e as técnicas e respalda uma concepção de formação profissional que unifique ciência, tecnologia e trabalho, bem como atividades cognitivas e instrumentais. A partir do pressuposto de indissociabilidade entre teoria e prática na formação dos estudantes, o PPI apresenta como compromisso institucional o desenvolvimento de competências profissionais conforme se observa na sequência:

\begin{abstract}
A UTFPR entende que competência não se limita ao saber fazer, pois pressupõe acerto no julgamento da pertinência da ação e no posicionamento, de forma autônoma, do indivíduo diante de uma situação. A ação competente envolve atitude relacionada com a qualidade do trabalho, a ética do comportamento, a responsabilidade social, o cuidado com o meio ambiente, a convivência participativa e solidária, iniciativa, criatividade, entre outras. E, assim sendo, por sua natureza e características, a Educação Tecnológica deve contemplar o desenvolvimento de competências profissionais tecnológicas, gerais e específicas, incorporando os fundamentos científicos, humanísticos e ambientais, necessários ao desempenho profissional e a uma atuação cidadã (PPI, 2019, p.25).
\end{abstract}

Alcançar competências profissionais, neste sentido, pressupõe muito mais que a apropriação de um conhecimento científico. Pressupõe a necessidade do estudante mobilizar e integrar esses conhecimentos em situações de uso realistas situadas em um contexto social, cultural, econômico e científico que permeiam a vida profissional e portanto também devem permear o processo de formação. Este é o cenário institucional que motivou a busca de fundamentos para estruturar o currículo do Curso de Engenharia Civil da UTFPR, campus Guarapuava, em uma perspectiva cognitivista voltada ao desenvolvimento de competências.

Diante do exposto, o objetivo deste trabalho é apresentar as estratégias que foram adotadas na reformulação do currículo do Curso de Engenharia Civil da UTFPR, campus Guarapuava, bem como a nova configuração da disciplina de Cálculo I, apresentada como forma de exemplificar como serão as disciplinas a partir da reestruturação curricular. 


\section{REVISÃO TEÓRICA}

O currículo de curso expressa inevitavelmente seus valores e concepções assumidos pela instituição em sua organização. A mudança de paradigma sobre a concepção de como o estudante aprende e o que se espera formação dos estudantes impõe a estruturação de um currículo integrado entendendo que,

O currículo disciplinar é entendido como consequência de princípios de organização curricular baseados na lógica das ciências ou na natureza do conhecimento, enquanto currículos integrados são entendidos como algo baseado nos interesses e necessidades dos alunos e na relevância social do conhecimento (LOPES, 2008, p.43).

Na perspectiva adotada pela autora o currículo integrado pode ser resultado de diferentes perspectivas teóricas, expresso em matrizes diferenciadas, mas a valorização das experiências dos estudantes se constitui como princípio integrador. Considerando o propósito da educação superior na qual a vinculação entre trabalho e educação define um programa formativo sistemático que proporciona o desenvolvimento do estudante a construção do currículo por competências, conforme preconizado seja nos parâmetros apresentados pela escola francesa ou britânica, apresenta-se como uma possibilidade real de integração.

O conceito de competência construído no cenário teórico educacional apresenta-se de forma bastante polissêmica, entretanto devido à abrangência da proposição realizada por Rogiers (2000), assume-se na UTFPR, que "A competência é um saber-agir baseado na mobilização e utilização interiorizadas e eficazes de um conjunto integrado de recursos, tendo em vista resolver uma família de situações-problema" (Rogiers, 2000, p.66).

Ao mapear o conceito de competência é possível perceber que esse conceito agrega três tipos de saberes: o saber, o saber-fazer e o saber ser. O saber relaciona-se aos conhecimentos que são historicamente legitimados como objeto de estudo e que já fazem parte dos currículos acadêmicos. O saber-fazer refere-se a um agir, seja de forma motora ou cognitiva, que está pautado na mobilização, integração e transferência de conhecimentos para diferentes situações. A integralização da competência se alcança com o saber-ser entendida como as características dos estudantes, que podem ser objetivos da formação a serem atingidos, como, por exemplo, características pessoais que devem ser atingidos previamente para uma aprendizagem ou ainda como critérios de qualidade particulares a certos desempenhos (Scallon, 2015).

Por tratar-se de competências complexas nas quais o estudante precisa saber agir mobilizando, integrando e transferindo conhecimentos, o tempo e os esforços dispensados para alcançá-las precisam ser cuidadosamente planejados no âmbito curricular para evidenciar as etapas de aprendizagem que serão percorridas e visualizar o papel das disciplinas/áreas de conhecimento no processo de ensino. Essas etapas foram apresentadas na Matriz Curricular como Elementos de Competência que serão desenvolvidos por um conjunto de disciplinas que assumem diferentes responsabilidades em desenvolver um conjunto de elementos de uma competência e de mais que uma competência. Baseado nas contribuições de Le Boterf (2015), quanto às operações cognitivas realizadas pelos estudantes utilizando os conhecimentos, as disciplinas assumem diferentes papéis ao longo do curso. Elas podem ser internalizadoras, quando apresentam e internalizam conhecimentos, mobilizadoras quando o estudante é levado a recuperar conhecimentos que já dispõe, internalizados em outras disciplinas ou, ainda, conhecimentos que o cercam para ampliar e integrar na resolução de problemas. E por fim existem disciplinas certificadoras nas quais, por meio de uma disciplina, o estudante deverá 
mobilizar, integrar e transferir conhecimentos em diferentes situações que demonstrem que ele desenvolveu a competência, ou seja, é na disciplina certificadora que se conclui um ciclo formativo.

O currículo baseado por competência traz a centralidade no processo de aprendizagem e, especialmente, no papel do estudante nesse processo. Zabala (2014) aponta que a regulação da própria aprendizagem é fator chave para a aprendizagem de competências, pois exige do estudante o planejamento das estratégias de aprendizagem para cada situação, aplicá-las, controlar o processo e, ao final dele, avaliar a eficiência de suas escolhas para possíveis alterações. Para que se tenha condições de regulação e consequente autonomia em relação à própria aprendizagem é imprescindível a compreensão do que se pretende alcançar.

Nesse sentido, além da definição das competências e elementos de competências que se inscrevem na dimensão do macroplanejamento do projeto pedagógico de um curso, é necessário estabelecer os parâmetros almejados para o microplanejamento, relacionados à organização disciplinar, denominados resultados de aprendizagem. Os Resultados de Aprendizagem "São declarações, do ponto de vista do estudante, indicando o nível cognitivo e de desempenho que se espera que o estudante alcance como resultado do engajamento na experiência de ensino e aprendizagem" (BIGGS; TANG, C, 2007). Ainda, possibilitam ao estudante traçar os próprios percursos de aprendizagem e também indicam de forma objetiva como as disciplinas se integram na formação das competências do engenheiro civil.

A definição do nível cognitivo e de desempenho que compõem os resultados de aprendizagem apoiam-se na Taxionomia de Bloom (1956), revisada por Anderson et al (2001), que apresenta as dimensões do processo cognitivo em uma crescente de complexidade na qual a primeira categoria se inscreve na dimensão de lembrar, seguida por compreender, aplicar, analisar, sintetizar e, por fim, o mais alto nível cognitivo na dimensão, Criar.

\section{METODOLOGIA}

\subsection{Metodologia de trabalho}

Os trabalhos iniciaram com a participação de membros do Núcleo Docente Estruturante (NDE) e da pedagoga do campus em uma Oficina de Design de Cursos com vistas a instrumentalizar de forma teórica e prática a construção de uma nova matriz curricular, baseada no desenvolvimento das habilidades e competências expressas nas novas diretrizes. Nesse curso, em linhas gerais, foram construídos o perfil do egresso desejado pelo curso, as competências a serem desenvolvidas e os elementos que as constituem, para o curso de Engenharia Civil da UTFPR campus Guarapuava.

O processo de atribuição de disciplinas aos Elementos de Competência (EC) foi realizado em encontros presenciais com os professores do NDE, colegiados e professores convidados. O resultado foi a alocação das disciplinas nos períodos do curso, observando não exceder a carga horária semanal de 30 horas/aula. Destaca-se que foi dispensada especial atenção aos primeiros períodos, de forma a possibilitar a integração dos estudantes ao curso e aos últimos períodos para assegurar a possibilidade de realização de estágio.

Após isso, em virtude pandemia da COVID-19, uma nova organização de trabalho foi imposta aos professores que passaram a trabalhar em plataforma digital. Nesse cenário, os professores das diferentes áreas que compõem o curso reuniram-se para transformar as ementas das disciplinas em temas de estudo (TE). A partir da construção dos TEs e da observação dos ECs aos quais a disciplina contribuí, os resultados de aprendizagem (RAs) foram construídos. O aprimoramento dos TEs e dos RAs foi realizado de forma coletiva em 
encontros do NDE, com a participação de todos os professores das áreas específicas do curso e da pedagoga.

\subsection{Metodologia De Elaboração Da Matriz Curricular Do Curso De Engenharia Civil Do Campus Guarapuava}

\section{O perfil do Egresso}

O trabalho no campus assumiu o perfil do egresso construído na Oficina de Design de Curso, por representantes do curso de engenharia civil da UTFPR - Campus Guarapuava considerando o contexto profissional e as demandas do mercado. O perfil construído pode ser observado na sequência:

"Os egressos do Curso de Engenharia Civil são profissionais que se caracterizam por criar soluções de engenharia para o desenvolvimento sustentável do ambiente construído, levando em consideração as demandas da sociedade. Poderão atuar na indústria da construção civil e em órgãos governamentais a ela associados. Para tanto, são capazes de projetar sistemas, produtos e processos relacionados à construção civil considerando a ética, a segurança, a legislação e os impactos ambientais; implementar obras de construção civil de forma responsável, eficaz e com liderança de equipes multiprofissionais; gerenciar organizações correlatas à indústria da construção civil e/ou recursos naturais, de forma empreendedora, inovadora e sustentável".

\section{As Competências do curso de Engenharia Civil}

A partir do perfil do egresso foram estabelecidas seis competências, sendo três delas de formação geral e três de formação específica do engenheiro civil:

- Investigar (Iv) problemas de contexto real integrando conhecimentos técnicocientíficos da literatura, selecionando informações relevantes e aplicando em soluções mais adequadas às contingências com integridade autonomia reflexiva e com senso crítico.

- Resolver (Rv) problemas estruturados de diferentes contextos da engenharia, de maneira autônoma, integrando os fundamentos de química, física e matemática, o raciocínio lógico e as ferramentas tecnológicas.

- Intervir (It) em situações conflituosas, fundamentado em conhecimentos da dimensão social, econômica, cultural, tecnológica e ambiental, demonstrando autonomia, comunicação qualificada, responsabilidade, autoria e autenticidade.

- Executar (Ec) projetos de construção civil, integrando a interpretação do projeto, o plano operacional e de viabilidade, a execução, o monitoramento retroalimentado e o replanejamento, com prioridade na utilização racional de recursos materiais, financeiros e tecnológicos, e na coordenação regulada de equipes multiprofissionais, de modo responsável, eficiente e cooperativo.

- Projetar (Pj) sistemas em diferentes contextos da construção civil, articulando adequadamente análise de demanda, diagnóstico de viabilidade, busca de referências, seleção de concepções e emprego de ferramentas tecnológicas, conforme os aspectos de funcionalidade, segurança e sustentabilidade, de forma crítico-reflexiva, criativa e com responsabilidade social.

- Gerenciar (Gr) diferentes organizações correlatas à indústria da construção civil, com visão sistêmica, empregando planejamento, metodologias e ferramentas gerenciais e tecnológicas devidamente validados para tomadas de decisão sustentáveis e 
inovadoras, com responsabilidade socioambiental, de forma empreendedora, colaborativa e reflexiva.

O estudante desenvolverá as competências ao longo dos períodos de curso, sendo que a primeira competência, Investigar, deverá ser completamente desenvolvida e, portanto, certificada, ao final do $4^{\circ}$ período do curso. As demais competências são certificadas nos períodos subsequentes, até o $9^{\circ}$.

A certificação é a conclusão do ciclo de aprendizagem no qual o estudante demonstra ter alcançado a competência que contempla o saber, o saber-fazer e o saber ser relativos ao desempenho e aos conhecimentos necessários para sua formação.

\section{RESULTADOS}

A disciplina de Cálculo I, tem 90 horas e está inserida no $2^{\circ}$ período do curso. No contexto de reformulação do projeto pedagógico do curso, para perspectiva de competências, buscou-se elaborar temas de estudo que representassem a reorganização dos conteúdos e estivessem diretamente vinculados ao desenvolvimento das competências solicitadas. No quadro 3 estão apresentados os nove temas de estudo definidos para esta disciplina. A carga horária total foi distribuída considerando a complexidade dos temas de estudo e, por conseguinte, o tempo de dedicação necessário à aprendizagem.

O comprometimento da disciplina com a perspectiva da formação por competências pode ser observado por meio da integração dos elementos de competência solicitados aos resultados de aprendizagem, que estabelecem a união entre o saber-fazer, o saber-ser e o conhecimento específico da disciplina (saber). Na figura 1 estão apresentados os elementos de competência que acionam a disciplina de Cálculo I e os resultados de aprendizagem construídos para os temas de estudo indicados no quadro anterior. Evidencia-se que a maioria dos ECs acionadores da disciplina de cálculo I fazem parte da competência Resolver (Rv), sendo que apenas o primeiro elemento de competência apresentado na figura compõem a competência Investigar (In).

Quadro 3 - Temas de estudo definidos para disciplina de cálculo

\begin{tabular}{||l|l||}
\multicolumn{1}{|c|}{ Tema de estudo } & $\mathrm{CH}$ \\
\hline $\begin{array}{l}\text { TE_1: Limite e continuidade: definição e propriedades para funções de uma } \\
\text { variável real a valores reais }\end{array}$ & $15 \mathrm{~h}$ \\
\hline TE_2: Derivadas: definição, propriedades e regras de derivação & $20 \mathrm{~h}$ \\
\hline TE_3: Aplicações de derivada: taxas de variação e máximos e mínimos & $10 \mathrm{~h}$ \\
\hline \hline TE_4: Esboço de gráficos de funções de uma variável real a valores reais & $5 \mathrm{~h}$ \\
\hline $\begin{array}{l}\text { TE_5: Primitiva de função de uma variável real a valores reais: definição e } \\
\text { propriedades }\end{array}$ & $5 \mathrm{~h}$ \\
\hline \hline TE_6: Integral de Riemann: definição e propriedades & $5 \mathrm{~h}$ \\
\hline \hline TE_7: Técnicas de integração & $15 \mathrm{~h}$ \\
\hline TE_8: Aplicações de integral: cálculo de área, volume, comprimento de curvas & $10 \mathrm{~h}$ \\
\hline \hline TE_9: Integrais impróprias & $5 \mathrm{~h}$ \\
\hline \hline
\end{tabular}


Figura 1 - Elementos de competência, temas de estudo e resultados de aprendizagem da disciplina de cálculo I Analisar resultados de validação, replanejando método, estratégia ou procedimento de forma reflexiva

\begin{tabular}{l|l|l|r} 
TE_1 & TE_2 & TE_3 & TE_4
\end{tabular}

RA_1: Avaliar criticamente esboços de gráficos de funções, considerando crescimento, concavidade e comportamento assintótico

Interpretar os problemas estruturados no contexto das Engenharias TE_4

RA_2: Interpretar problemas com informações de gráficos de funções de uma variável real, extraindo adequadamente os dados necessários ao desenvolvimento da solução

Delimitar problemas interpretados identificando as variáveis e as condições de contomo

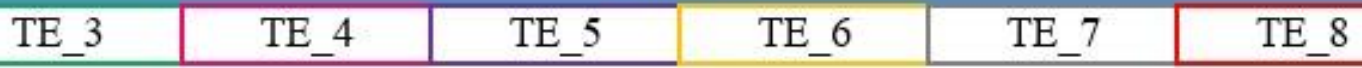

RA_3: Descrever problemas interpretados fazendo bom uso do conceito de taxas de variação e de aplicações de integral, com autonomia

\begin{tabular}{|c|c|c|c|c|c|c|c|c|}
\hline \multicolumn{9}{|c|}{ Elaborar hipóteses plausíveis aos problemas delimitados } \\
\hline TE_1 & TE 2 & TE_3 & TE_4 & TE_5 & TE 6 & TE 7 & TE_ 8 & TE_9 \\
\hline
\end{tabular}

RA_4: Analisar com criticidade as situações propostas por meio dos conhecimentos de limite, derivada e integral, obtendo caminhos plausíveis para a resolução do problema dado

Determinar a solução por meio de linguagem matemática e raciocínio lógico,

empregando ferramentas tecnológicas adequadas à hipótese

\begin{tabular}{l|l|l|l|l|l|l|l|l} 
TE_1 & TE_2 & TE_3 & TE_4 & TE_5 & TE_6 & TE_7 & TE_8 & TE_9 \\
\hline
\end{tabular}

RA_5: Resolver corretamente problemas de funções de uma variável real, considerando o cálculo de limites, técnicas de derivação e integração, com autorregulação

\section{Replanejar o processo, caso a hipótese não seja validada}

\begin{tabular}{|c|c|c|c|c|c|c|c|}
\hline TE_2 & TE_3 & TE_4 & TE_5 & TE_6 & TE_7 & TE_8 & TE 9 \\
\hline
\end{tabular}

RA_6: Analisar a adequação da solução obtida aos pressupostos do problema, readequando as ferramentas utilizadas de derivação e integração na reformulação da solução, de forma reflexiva

A construção apresentada demonstra o registro documentado de um processo amplo de discussão curricular que abrange a mudança de paradigmas na formação dos engenheiros. $\mathrm{O}$ envolvimento dos professores em todo processo teve como premissa a compreensão coerência entre a proposta curricular e a prática pedagógica de cada professor para que os estudantes tenham oportunidades de desenvolver plenamente as competências propostas. 


\section{CONSIDERAÇÕES FINAIS}

No exposto, constatam-se avanços relacionados à integração dos resultados de aprendizagem de cada disciplina ao perfil do egresso e às competências desejadas, especialmente no compromisso com o desenvolvimento do saber-fazer e do saber-ser. Contudo, o novo modelo impõe desafios quanto à continuidade da formação dos professores para implementação do currículo, especialmente para sanar as preocupações relacionadas ao processo avaliativo que assegure coerência com os resultados de aprendizagem esperados. Nesse sentido, entende-se a necessidade concentrar esforços na formação de professores e, também, na mudança das condições estruturais da universidade que viabilizem as novas metodologias de ensino e avaliação.

Outra consideração importante versa sobre a relevância do trabalho coletivo, reflexivo, colaborativo e integrado no processo de reformulação do PPC, o que aponta para a necessidade de que este formato de trabalho seja replicado na consolidação do currículo proposto.

Por trata-se de um processo ainda em construção, com mudanças significativas no currículo do curso, entende-se que será imperativo o acompanhamento continuo da implementação. Os quantitativos e qualitativos relacionados à percepção dos professores e estudantes levantados no decorrer do acompanhamento subsidiará os ajustes, alteração e melhoramentos que se façam necessários e também a análise mais aprofundada em registros posteriores.

\section{Agradecimentos}

Os autores agradecem ao DEPEDUC/UTFPR pela realização da Oficina de Design de Curso e aos professores do Colegiado e do Núcleo Docente Estruturante do curso de Engenharia Civil da UTFPR-Guarapuava pela dedicação e compromisso ao longo do processo de discussão e reformulação do PPC.

\section{REFERÊNCIAS}

ANDERSON, Lorin W., KRATHWOHL, David R. AIRASIAN, Peter W. A taxonomy for learning, teaching and assessing: a revison of Bloom's Taxonomy of Educational Objectives. Nova York: Addison Wesley Longman, 2001. 336 p

BRASIL. Conselho Nacional de Educação. Resolução CNE/CES no 02/2019, de 24 de abril de 2019. Institui as Diretrizes Curriculares do Curso de Graduação em Engenharia. Diário Oficial da União, Brasília, 26 de abril de 2019, Seção 1, pp. 43 e 44.

BIGGS, John, TANG, Catherine. Using Constructive Alignment in Outcomes-Based Teaching and Learning Teaching for Quality Learning at University (3rd ed., pp. 50-63). Maidenhead: Open University Press, 2007

LE BOTERF, Guy. De la compétence: Essai sur sun un attracteur étrange. Paris: Les èdition d'Organisation. 1994.

LOPES, Alice Casimiro. (2008). Políticas de integração curricular. Rio de Janeiro: UERJ.

ROGIERS, Xavier. Une pédagogi de l'intégracion: Compétences et intégracion des acquis dans l'enseignement. Bruxelles: De Boeck Iniversité. 2000. 
"Os desafios para formar hoje o engenheiro do amanhã"

SCALLON, Gérard. Avaliação da aprendizagem em uma abordagem por competências. Tradução de Juliana Vermelho Martins. Curitiba: PUCPRess, 2015.

UTFPR, Projeto Pedagógico Institucional (PPI) da Universidade Tecnológica Federal do Paraná, Curitiba, 2019.

ZABALA, Antoni. Como aprender e ensinar competências [recurso eletrônico] / Antoni Zabala, Laia Arnau; tradução: Carlos Henrique Lucas Lima ; revisão técnica: Maria da Graça Souza Horn. - Porto Alegre: Penso, 2014. E-PUB Editado como livro impresso em 2010.

\title{
THE CURRICULUM OF THE CIVIL ENGINEERING COURSE FOCUSED ON LEARNING: THE COMPETENCE MATRIX AS A POSSIBILITY OF INTEGRATION
}

\begin{abstract}
After the publication of the National Curriculum Guidelines (DCN) for engineering courses, in 2019, higher education institutions (HEIs) sought to adapt to the new organization. To reformulate the pedagogical projects of the engineering courses, the Federal Technological University of Paraná (UTFPR), through the Department of Education, organized a Workshop of Course Design. The objective of this workshop was to develop the pedagogical projects in order to ensure the development of the necessary skills for the engineering professional. With the training process, the Structuring Teaching Nucleus (NDE) and the teachers of the Civil Engineering course, campus Guarapuava, built a new curricular proposal, in order to guarantee technical training and the development of general and specific skills. Teachers worked collectively in the review of all course subjects, seeking to adapt them to the learning model of the three types of knowledge: know (knowledge), knowhow to do (technical knowledge) and know-how to be (attitude). This review started by "transforming" the contents into study subjects (TE), which refer to knowledge, making it more attractive to the student. From the TEs, the group elaborated the learning results (AR), which link the three types of knowledge to the construction of the graduate profile. In this work, the skills and elements necessary to form the profile of the graduate of the Civil Engineering course at UTFPR, campus Guarapuava, are presented, and the TEs and RAs of the Calculus I discipline are presented as an example.
\end{abstract}

Keywords: Competency Teaching. Civil Engineering. Calculus. 